\title{
Blast Waves and How They Interact With Structures
}

\author{
IG Cullis
}

\begin{abstract}
The paper defines and describes blast waves, their interaction with a structure and its subsequent response. Explosions generate blast waves, which need not be due to explosives. A blast wave consists of two parts: a shock wave and a blast wind. The paper explains how shock waves are formed and their basic properties. The physics of blast waves is non-linear and therefore non-intuitive. To understand how an explosion generates a blast wave a numerical modelling computer code, called a hydrocode has to be employed. This is briefly explained and the cAst Eulerian hydrocode is used to illustrate the formation and propagation of the blast wave generated by a $1 \mathrm{~kg}$ sphere of TNT explosive detonated $1 \mathrm{~m}$ above the ground. The paper concludes with a discussion of the response of a structure to a blast wave and shows that this response is governed by the structures natural frequency of vibration compared to the duration of the blast wave. The basic concepts introduced are illustrated in a second simulation that introduces two structures into the blast field of the TNT charge.
\end{abstract}

\section{Introduction}

In both the military and civilian worlds, blast waves and their interactions with a structure need to be understood. Explosions form blast waves. These explosions can be either deliberate or accidental. Deliberate explosions can include demolition charges, weapon systems and improvised explosive devices. Accidental explosions can result from the ignition of gas clouds and inflammable liquids and chemicals due to fire or the failure of pressurised containers. In all cases the effects of the blast waves produced need to be quantified, particularly in respect of injuries to personnel.

A blast wave is formed by a sudden release of energy. The source of the energy release is not important. However, for many materials, particularly explosives, the very high rate of the energy release generates a tremendous power source. It is this power source that is responsible for the catastrophic damage often associated with an explosive.

The generation, development and propagation of blast waves are governed by the non-linear* physics that describes shock waves. In the case of an explosive, its' detonation properties define the properties of the blast wave. In the military scenario, blast waves created by explosive charges are the major concern. In this paper, therefore, the detonation of $1 \mathrm{~kg}$ of TNT is used to illustrate the main features of a blast wave interacting with structures - the ground and spherical objects.

Since blast waves are non-linear the response of a structure can also be highly non-linear. As will be discussed below the characteristics of an explosion, which have the most influence on structural response, are its peak pressure, impulse, and overall shape. The elastic-plastic strength and natural period of oscillation of the structure being loaded then determines the type of interaction and the response.

Before discussing blast waves, it is useful to understand the size and scope of the energy sources that generate blast waves and the non-linear physics that results. This has important consequences for the properties of blast waves and their propagation. The driving mechanism is the shock wave and a basic understanding of their formation and behaviour is essential for those interested in blast waves and their interaction with structures, including personnel.

\section{Explosions and Explosives}

An explosion is the phenomenon that results from a sudden release of energy. The source of the energy release may come from explosives such as gunpowder or TNT, from a chemical explosion, from wheat flour dust, from pressurized steam in a boiler, from a mechanical explosion or from an uncontrolled nuclear transformation.

Explosions are thus not restricted to chemical explosions. For example, a steam boiler may explode because of the heat energy which has been put into the water in the boiler. The thermal energy, however, is not intrinsic to the water, and water is not an explosive. The greatest steam explosion in
Technical Leader

Numerical Modelling

Defence Research and

Evaluation Agency,

Fort Halstead,

Sevenoaks,

Kent, TN14 7BP
*Imagine hanging a mass on the end of a wire. As we add more mass the wire stretches. If we add twice the mass the wire stretches by twice as much. Remove the mass and the wire returns to its former length. This is a linear process. If, however, the mass added exceeds the strength of the wire then it becomes plastic and continuously stretches until it breaks. This is a non-linear process. 


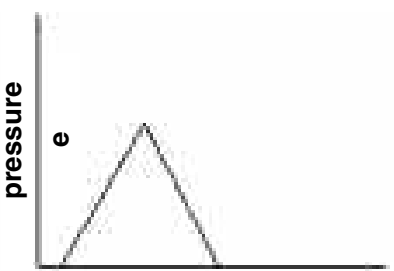

A. initial pulse

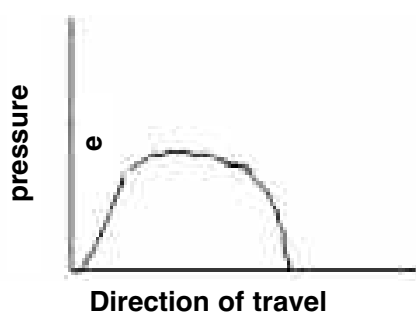

B. intermediate

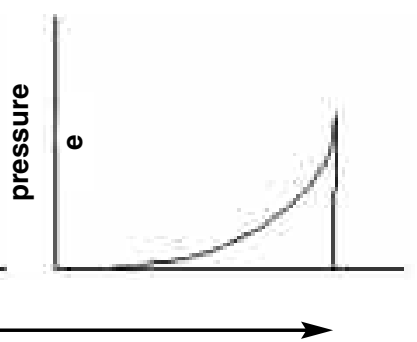

C. final shock phase

Fig 1. Development of a Shock Wave from an Initial Pressure Distribution in Air.

history was the famous Krakatoa volcanic explosion of 27 August 1883. It was caused by the sudden vaporization of an estimated 1 cubic mile of ocean water when a volcano ruptured and spilled a great mass of molten lava into the ocean. The blast wave was heard at a distance of 3000 miles. It has been estimated that this great explosion involved an energy release possibly equivalent to more than 5 billion tons of TNT.

The energy release must be a sudden one, rapid enough to cause a localisation of energy. This localised energy is then dissipated by a blast wave, propulsion of fragments and surrounding material, and in many cases thermal radiation.

An explosive is defined as a material which is capable of producing an explosion by its own energy. All explosives possess this single attribute. All explosives produce heat and nearly all produce gas. The gas is the primary mechanism by which an explosive does work on its surroundings.

High performance explosives possess the ability to release their energy over a very small time period. The energy release rate is governed by the Detonation Velocity, which for high performance explosives is of order $8 \mathrm{~mm} / \mu \mathrm{s}$ (millimetres per microsecond), or $8 \mathrm{~km} / \mathrm{s}$. This should be compared to the speed of sound in air at $0.33 \mathrm{~mm} / \mu \mathrm{s}$, $0.33 \mathrm{~km} / \mathrm{s}$. The importance of the speed of energy release can be illustrated by comparing the energy released by burning coal and the high performance explosive TNT - coal has greater available energy (Table 1).

Table 1: Comparison of energy release of coal and TNT

\begin{tabular}{lll}
\hline & $1 \mathrm{~kg}$ Coal & $1 \mathrm{~kg}$ TNT \\
\hline Energy Available & $24 \mathrm{MJ}$ & $4 \mathrm{MJ}$ \\
Time to Release the Energy & $2000 \mathrm{~s}$ & $10 \mu \mathrm{s}$ \\
Power Source & $12 \mathrm{KW}$ & $400,000 \mathrm{KW}$ \\
\hline
\end{tabular}

Thus $1 \mathrm{~kg}$ of $\mathrm{TNT}$, in terms of its power, is equivalent to 400 power stations generating at $1000 \mathrm{MW}$ for the same few microseconds. This is equivalent to the total electricity generating capability of the United States in 1979.

A sheet of TNT explosive $20 \mathrm{~m}$ square operates at a power level equal to all the power the earth receives from the sun. Close to a detonating explosive therefore, there can be a significant thermal radiation dose, which can result in an additional damage mechanism.

In terms of its ability to do work on its surroundings through the gaseous products, $1 \mathrm{~kg}$ ofTNT has an energy potential of 7MJ, almost twice its chemical energy.

With these perspectives in mind, it is readily apparent why relatively small amounts of explosives can cause such catastrophic damage.

\section{Shock waves}

A disturbance in a medium travels at the local speed of sound in that medium. In air, under normal conditions, a disturbance (e.g. a sound wave) travels with a speed of 330 $\mathrm{m} / \mathrm{s}$. In a metal, however, the same sound wave will travel with a speed of $5000 \mathrm{~m} / \mathrm{s}$. The speed of sound is a function of the local pressure and temperature. Therefore, if the pressure or temperature increases, the speed of sound also increases. This has a dramatic influence on the propagation of a pressure pulse of arbitrary shape and finite amplitude through the medium. Consider an idealised triangular pulse as shown in Figure 1A. Since each individual portion of the pulse has a different pressure, the local sound speed for each portion is different. Thus each region of the pulse travels with this local sound speed. The higher-pressure regions thus move faster than the preceding lower pressure regions. They catch up with these slower moving regions and the wave profile becomes steeper, as shown in Figure 1B. This process continues until in the limit, a sharp discontinuity is formed, Figure 1C. This is called a shock wave. The velocity of a shock wave is supersonic relative to the undisturbed medium into which it is travelling. Examples of shock waves in air include the sonic boom from a supersonic aircraft and the 'bang' one hears when a balloon is burst or an explosive charge detonated. A shock wave is an integral part of a blast wave and heralds its approach.

From the perspective of an observer at rest in the undisturbed medium, the arrival of a shock wave is characterised by an abrupt acceleration, a sudden jump in pressure and density and a local rise in temperature.

As a shock wave is supersonic compared to the local sound speed in the surrounding medium it is often convenient to describe it by a quantity called the MACH Number. 
The Mach Number (M) is the ratio of the shock speed to the local speed of sound, usually under ambient conditions.

Shock waves undergo reflection from surfaces in the same way as sound or light waves. However, unlike sound waves, where their effect on the properties of the medium is negligible, shock waves change the medium through which they are travelling. Their reflection from a surface is therefore very complex and non-intuitive. Shock waves exhibit three kinds of reflection phenomena:

- Normal reflection associated with head-on impact with a non yielding surface;

- Oblique reflection associated with a small angle of incidence with a surface;

- Mach stem formation, a spurt-type effect associated with angles of incidence with a surface near grazing incidence.

In the case of a normally reflecting sound wave from a rigid surface, the pressure doubles on reflection. However in the case of shock waves the reflected pressure is a nonlinear function of the Mach number $(M)$ of the incident shock wave. Thus if we consider a sound wave to be a very weak shock we can say for low Mach numbers $(M \sim 1)$ the reflection coefficient (the ratio of the reflected to the incident pressure) is 2 . For very strong shock waves in air, the theoretical upper limit of the reflection coefficient is 8 .

In the case of oblique reflection, the incident shock wave impinges upon a surface with a small angle of incidence and a shock wave is reflected back into the flow, Figure 2. In this respect they resemble sound waves. However, in general, unlike a sound wave the angle of reflection does not equal the angle of incidence.

A shock front impinging on a surface near grazing incidence does not reflect directly, but is deflected so that it spurts along the surface. As the angle of incidence increases and exceeds $40^{\circ}$ the flow travels parallel to the surface with the shock front perpendicular to the surface. This is called a Mach Stem. This surface shock extends from the surface out into the flow until it connects with a line of intersection between the incident shock and the reflected shock. The reflected shock is thus detached from the surface. This is shown schematically in Figure 2.

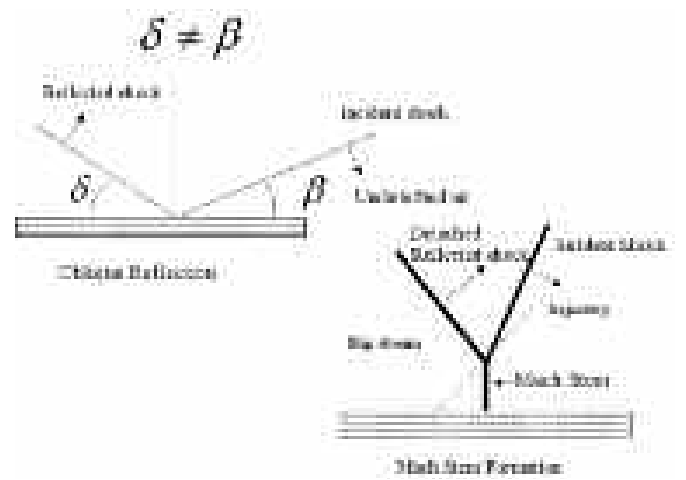

Fig 2. The Oblique Reflection and Mach Stem Regimes for Reflected Shock Waves.
This Mach stem regime, as will be shown below, is very important in the behaviour of blast waves. The most important feature is the direction of the blast wind behind it, which is parallel to the surface and travelling with a much higher velocity than in the incident wave.

This brief overview of shock waves and their behaviour is essential for an understanding of the formation and behaviour of blast waves. Given the nonlinearity of their behaviour, their interaction with a structure can have quite dramatic consequences, particularly in the case of personnel vulnerability.

\section{Detonation}

An explosive is a chemical compound that has energy locked up within its molecules and molecular bonds. When these chemical bonds are broken, energy is released since the solid explosive has a higher energy than the gaseous products produced by the reaction. The reaction is said to be exothermic. The reaction rate for such chemical reactions is an exponential function of temperature. Therefore if the temperature of the local surroundings increases, the rate of production of heat increases exponentially. This is much faster than the heat loss to the surrounding material, through heat conduction, which is linear. As the temperature rises there comes a point where heat evolution becomes the dominant process and rapid acceleration of the chemical decomposition reaction occurs.

A pressure wave associated with this chemical reaction is created, which propagates out into the explosive. The pressure wave compresses the explosive and heats it up, as do the hot gases generated by the chemical reaction. The higher temperature then increases the rate at which the chemical reactions occur, which drives the pressure wave even faster. This 'runaway' process continues with the pressure wave becoming steeper and steeper until it suddenly assumes a step condition, where the pressure, density and temperature suddenly jump from the initial state to a compressed state. As described above, this jump is just another example of a shock wave. The resultant heating of the solid explosive caused by the shock wave initiates the chemical reaction almost immediately. The reaction zone in which the chemical decomposition occurs is then physically located at the shock front and supports and drives it. The shock front travels at a supersonic velocity with respect to the unreacted explosive. For a high performance explosive, the reaction zone is less than $1 \mathrm{~mm}$ thick.

A shock wave driven by a chemical reaction is called a detonation wave. It is supersonic with respect to the unreacted, or 
undetonated, explosive. When initiated by a detonator the detonation wave is formed within a few microseconds and over a distance of about $2-10 \mathrm{~mm}$.

For a high performance explosive, the detonation velocity is typically of order $8 \mathrm{~km} / \mathrm{s}$. At the front of the detonation wave the pressure is about $20-30 \mathrm{GPa},(200-300$ kbar, where $1 \mathrm{kbar}$ is 1000 times atmospheric pressure). The temperature in the gaseous products immediately behind it can reach $7000^{\circ} \mathrm{C}$.

\section{Formation and propagation of blast waves}

To illustrate the blast wave generated by an explosion it is useful to consider the blast wave generated by $1 \mathrm{~kg}$ of TNT high explosive $50 \mathrm{~cm}$ above the ground. The shock waves produced can therefore be expected to be strong and governed by the non-linear physics associated with their formation and propagation. The linear physics of acoustics is inadequate to understand the resultant explosion.

To provide a detailed description of the physics of blast waves, we have to employ sophisticated numerical modelling techniques contained in a Hydrocode. The name hydrocode is used because they were first used to solve problems in hydrodynamics.

\section{Hydrocodes}

In its simplest form a hydrocode represents the problem within a numerical mesh and uses the physical properties of the materials in the problem to solve the conservation laws

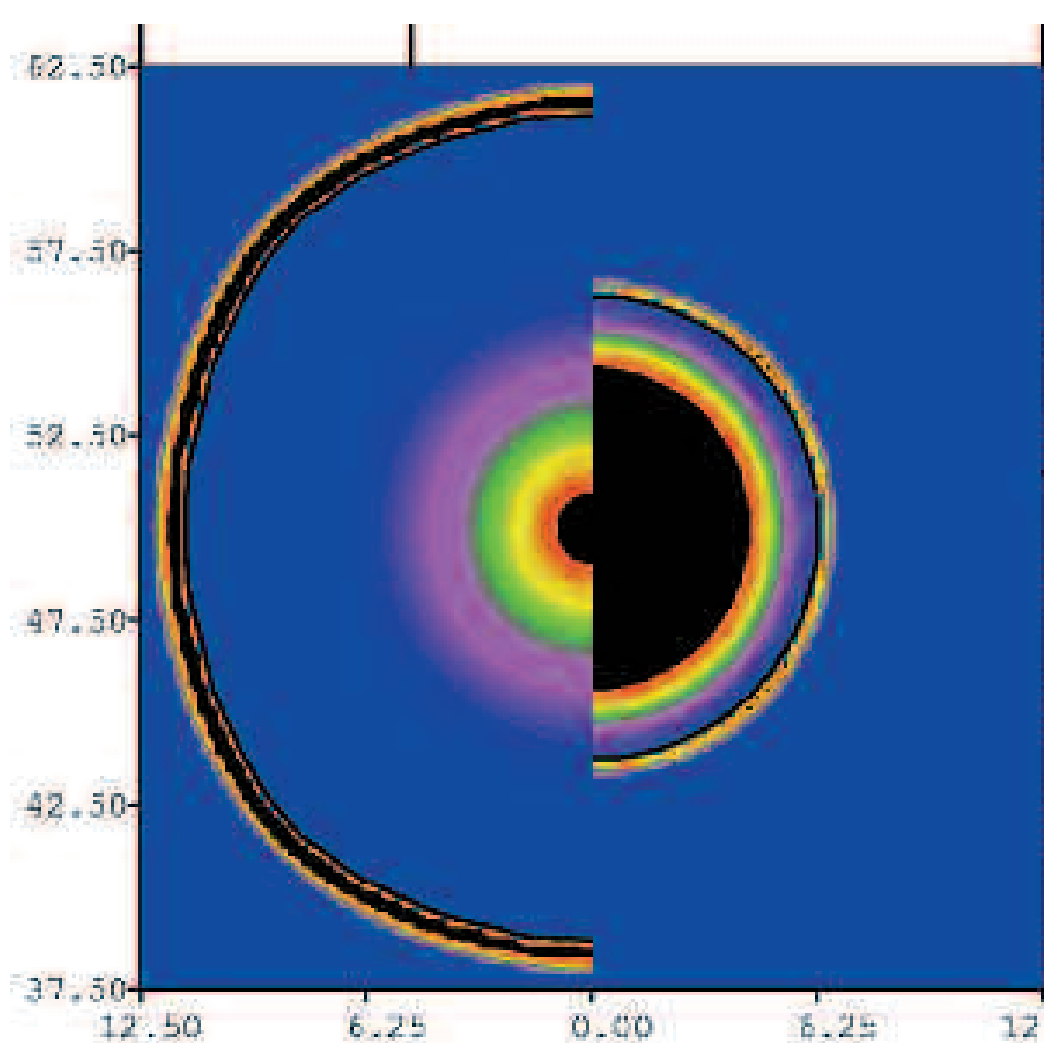

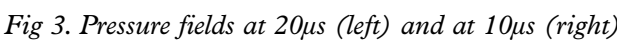

of mass momentum and energy (Newton's Laws) in each of the cells within the numerical mesh. This process calculates the pressure, energy, density and velocity of the flow field and advances the solution by a small time-step, of order one hundredth of a microsecond. By repeating this process many thousands of times, the explosive event is advanced in time.

There are two main numerical methods depending on the way in which the numerical mesh is defined. If the numerical cell is defined as being embedded in the material and deforming with it, then the simulation is a LAGRANGE simulation. If, however, the mesh is fixed in space and material moves through it, then the simulation is a EULERIAN simulation.

The Lagrange approach is generally used for problems with little significant deformation where material interfaces are important e.g. structural deformation. The Euler approach is generally used for problems characterised by significant material deformation and flow e.g. blast wave formation and propagation.

The Theoretical Modelling and Hydrocode Development group within the Guns and Warheads Department at DERA Fort Halstead has undertaken significant research over the past 20 years into the development and application of these numerical techniques to a wide range of problems in detonics and penetration mechanics. Central to this research is the integration of these simulations with smallscale controlled and highly instrumented experiments. This serves to validate the numerical and material models.

These modelling techniques have been assembled into a numerical toolbox called cAst (Computational Applied Science and Technology) to provide researchers, of any background, access to state of the art modelling capabilities.

\section{Free Field Explosions}

The Euler module within cAst has been employed to simulate the detonation of the 1 kg TNT charge, whose diameter is $11 \mathrm{~cm}$, and the formation of the blast wave, its development and subsequent interaction with the ground. Initially however, the charge can be considered to be a free field charge.

When a high performance explosive is detonated in air, the high pressure, high temperature detonation wave impinges on the explosive-air interface. The arrival of the detonation wave at the surface of the charge generates a shock wave in the air. Because air, a gas, is highly compressible, a thin layer is compressed by the detonation wave to a very high density, pressure and temperature. This temperature can reach $10000^{\circ} \mathrm{C}$.

The effects of this on the surrounding air can be understood from the hydrocode simulation. To assist this process an array of 


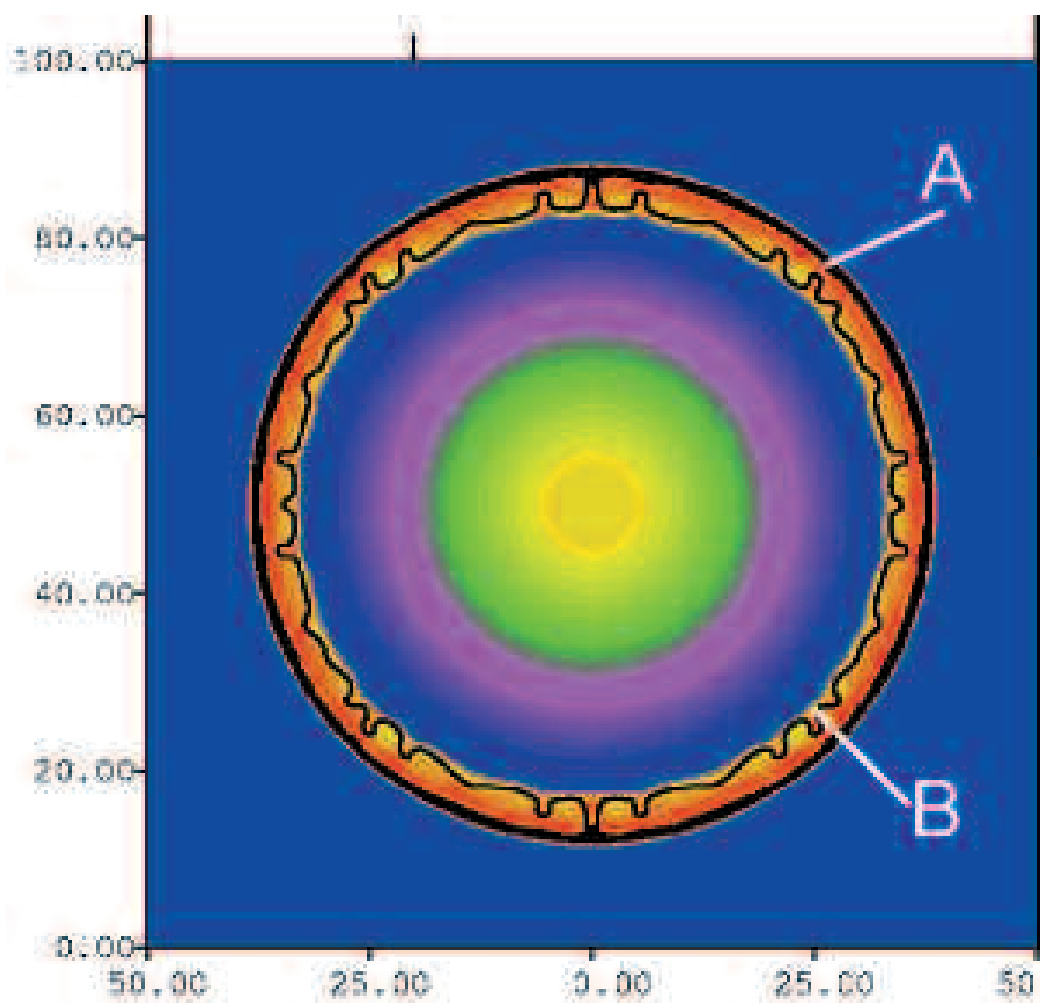

Fig 4. Pressure field at $100 \mu$ s. the product surface is supersonic with respect to the stationary air.

As the explosive products expand however, they cool down and their pressure, density, temperature and velocity fall. As a rule of thumb, for every doubling of their volume, their pressure falls by an order of magnitude. Once their velocity becomes sub-sonic with respect to the air, the shocked air layer detaches from the product cloud. The time it takes to do this is a function of the explosive and the charge size.

For our charge this process is welladvanced $100 \mu$ s after detonation, as shown in Figure 4. The orange 'rim' around the products is the heated air layer (A). The black line, with the regularly spaced protuberances is the interface between the air layer and the explosive products (B). Its shape reflects the turbulent mixing of the products and the air.

Most explosives have a chemical composition that is 'oxygen negative', i.e. the products formed by the detonation have not fully reacted with the oxygen in the air. As they mix they can continue to burn and release further energy. TNT is one of the most oxygen negative compositions and releases almost as much energy through this afterburning process as is released in the initial detonation. As will be explained below this can have a significant effect on the blast effects of a charge.

Thus, an observer close to the explosion will experience a high-pressure shock wave followed by a high-speed wind of decaying pressure due to the moving air and explosive products. This combination is called the blast wave.

We can see this process in the station pressure histories, shown in Figure 5.

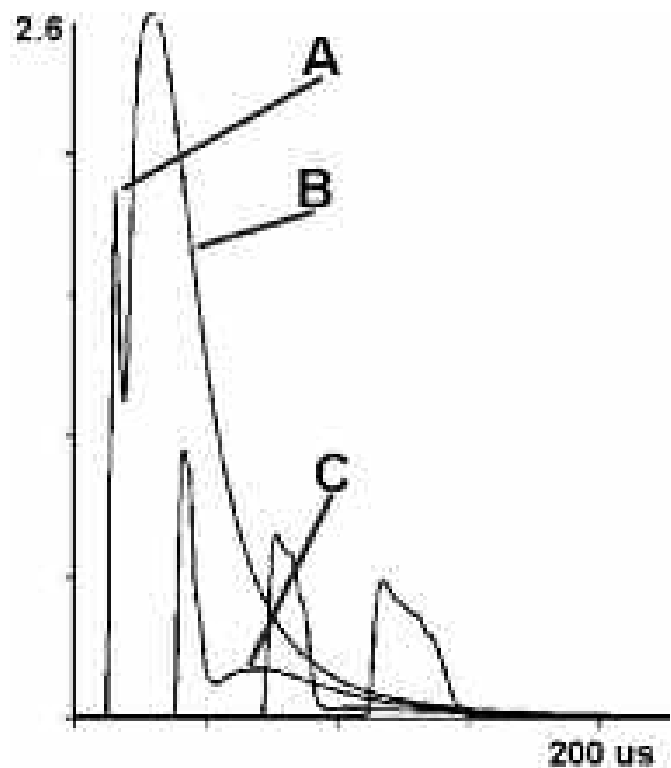

Fig 5. Pressure time histories for $1 \mathrm{~kg}$ spherical charge of TNT at 10,20,30(left) and $40 \mathrm{~cm}$ (right) from the charge centre.

The histories shown in the figure are for the stations $10,20,30$ and $40 \mathrm{~cm}$ from the centre of the charge. The time axis extends to $200 \mu$ s. The pressure axis extends to $2.6 \times 10^{8}$ explosive products, because the velocity of 
dynes $/ \mathrm{cm}^{2}$ i.e. 257 atmospheres.

The first history, $10 \mathrm{~cm}$ from the charge, shows the air shock, pressure about 170 bar (A), arriving at $15 \mu$ s followed at $30 \mu \mathrm{s}$ (B) by the explosive products, which have a much higher pressure of 257 atmospheres and a longer duration than the air shock.

The subsequent histories show a broadening of the blast wave as the air shock moves away from the products. The pressure initially falls quite rapidly and then more gradually as the wave expands away from the charge. The air shock, at the head of the blast wave, is clearly visible in all of the pressure histories. It reaches the second station, $20 \mathrm{~cm}$ from the centre of the charge, at $42 \mu$ s after detonation. The explosive products can still be distinguished in the pressure record (C), but their pressure has fallen dramatically.

The blast wave reaches the remaining two stations at $70 \mu \mathrm{s}$ and $117 \mu \mathrm{s}$ respectively. Although the products reach these gauge positions, their pressure is so low compared to the air, that they do not make a significant contribution to the blast wave, in terms of pressure.

This process continues until at larger distances the blast wave simply consists of the air shock and high-speed movement of air, since the products fall further and further behind it. At large distances from the charge it is the expansion of the shock wave and the rapidly moving air behind it that constitute the blast wave. The rapidly moving air behind the shock front is often referred to as the blast wind.

Close to the explosive charge, the thermal output (discussed in the section on explosives and explosions) is likely to ignite any surrounding combustible material. The blast wave will at the same time rapidly

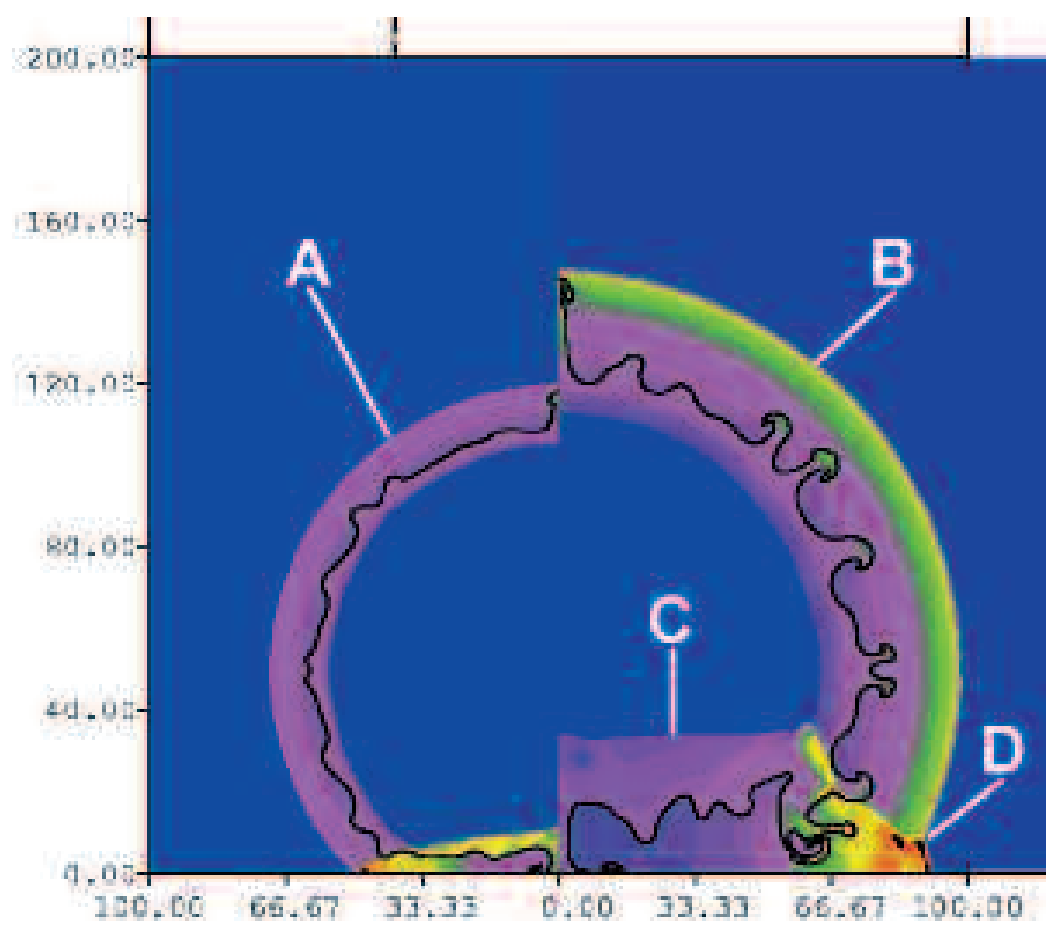

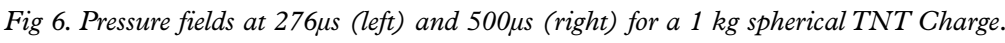

accelerate and disperse it.

In addition of course there is a transitory temperature rise associated with the passage of the air shock, which close to large explosions can be significant. Due to the irreversible nature of the shock process, there is also a permanent temperature change which can effect equipment and personnel.

\section{Ground Effects}

The discussion so far has concerned a spherical charge detonated in free field, i.e. the charge is far removed from an object or the ground. In reality, the ground and/or an object close by almost always has an influence on the propagation of a blast wave.

In the next simulation, the charge is $50 \mathrm{~cm}$ above the ground. The blast wave impacts the ground at about $200 \mu$ s (equivalent to an average blast wave velocity of $2000 \mathrm{~m} / \mathrm{s}$ ). At this point the shock front is approximately 6 $\mathrm{cm}$ ahead of the products. On impact with the ground the shock wave is reflected back into the products and the surrounding air. This acts to reinforce the blast pressure. The reflected pressure from the ground can therefore be between 2 and 8 times the incident pressure.

The propagation of the reflected shock wave through the fireball and the air shock is shown in Figure 6 in the pressure fields at $276 \mu$ s (left) (labelled A) and $500 \mu$ s (right) (labelled B). Notice how the reflected shock travels faster through the products than through the shocked air (C). This is because the temperature of the products is higher than the surrounding air and the speed of sound is therefore greater. This effect is even more pronounced for explosives that have significant afterburning, since they are at a higher temperature.

Also visible in the figure is the early formation and development of the Mach stem. The figure shows the triple point and its trajectory, (labelled D), idealised in Figure 2.

The figure also shows the effect the reflected shock has on the product fireball. The particle velocity, initially directed towards the ground is now reversed by the reflected shock. The fireball thus begins to rise.

The blast wave continues to propagate away from the explosion, with the reflected shock wave, because of its higher pressure and velocity, gradually overtaking it. The blast pressure fields at $900 \mu \mathrm{s}$ and $1.2 \mathrm{~ms}$ are shown in Figure 7 and at $4 \mathrm{~ms}$ in Figure 8.

By this time the reflected wave accounts for about two-thirds of the blast field. For an observer far enough away from the explosion, the total measured blast wave is this reflected wave. Notice also how the product cloud has rolled up into the classic 'mushroom' shape as it ascends into the atmosphere. This is a direct consequence of the processes described above and the reflected shock wave from the ground. 


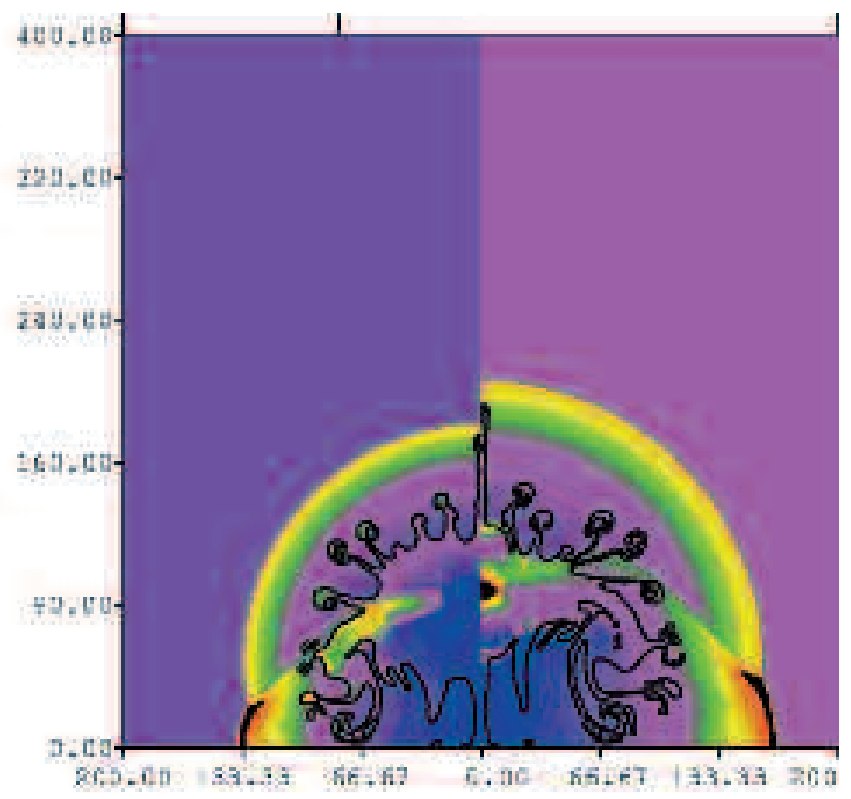

Fig 7. Pressure fields at $900 \mu \mathrm{s}$ (left) and $1.2 \mathrm{~ms}$ (right) for a $1 \mathrm{~kg}$ spherical TNT Charge.

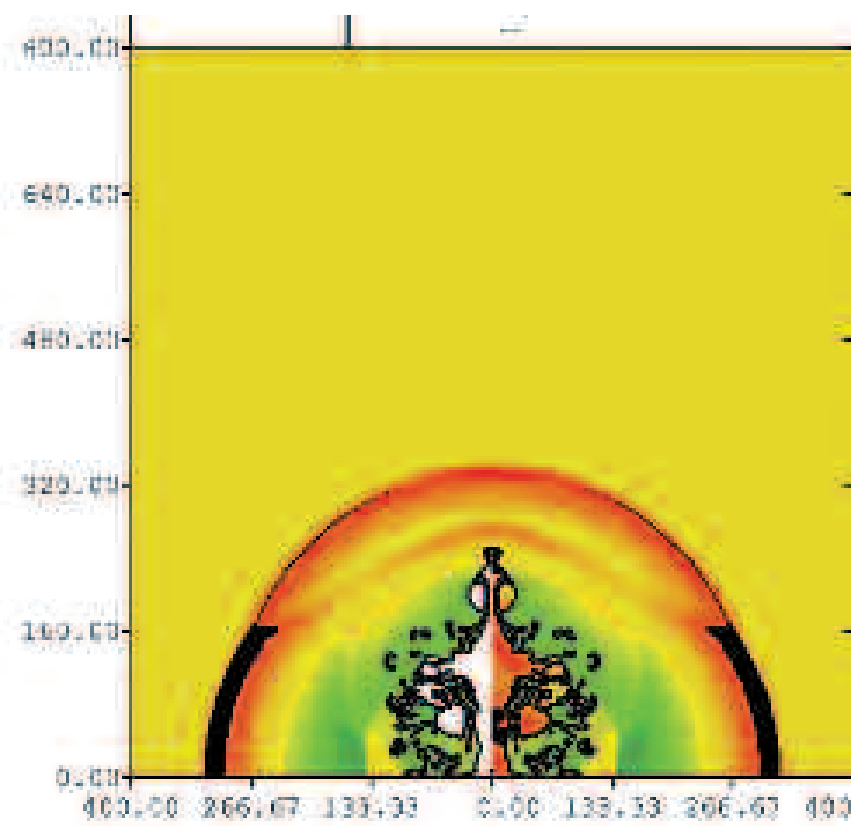

Fig 8. Blast Pressure Field at $4 \mathrm{~ms}$ after detonation of a $1 \mathrm{~kg}$ spherical charge of TNT $50 \mathrm{~cm}$ above the ground.

The effect of the reflected shock on an observer $1 \mathrm{~m}$ away from the centre of the charge and $50 \mathrm{~cm}$ above the ground is shown in the pressure history in Figure 9 (left). The history shows the arrival of the initial blast wave, with a shock pressure of 8.8 atmospheres, at $520 \mu$ s (labelled A) followed at $900 \mu$ s by the reflected shock wave from the ground (labelled B). This has the effect of raising the blast pressure, at this time interval, from 1.7 atmospheres to 3.4 atmospheres; i.e. the reflected shock doubles the pressure.

The pressure record also shows an additional feature due to the dynamics of the blast wind. The moving air and explosive products behind the shock front, that constitute the blast wind, continue to expand and their pressure therefore falls. The inertia in the flow however, means that the gases over-expand. The pressure falls below
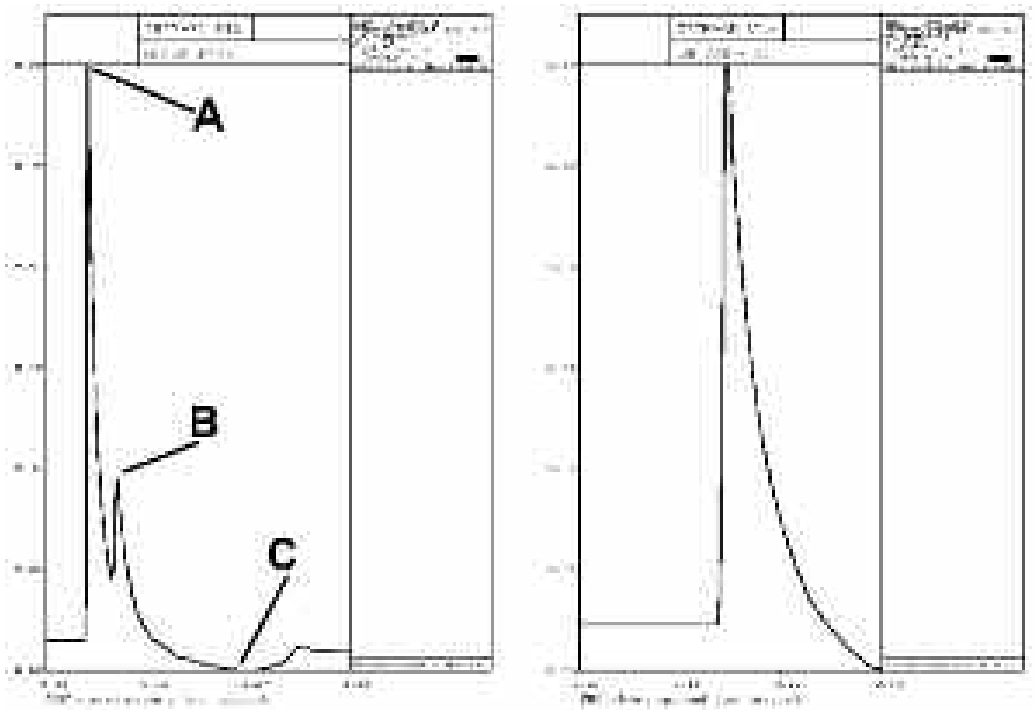

Fig 9. Pressure-time histories for observers $50 \mathrm{~cm}$ above the ground for a $1 \mathrm{~kg}$ spherical charge of TNT. Figure 9 (left): Observer $1 m$ from charge; Figure 9 (right): Observer $2 m$ from the charge. atmospheric, i.e. there is a 'negative' relative pressure phase associated with the tail of the blast wave (labelled C). This is clearly shown in Figure 9 (left) and has duration, at this point, of almost twice the initial positive phase. This negative pressure regime acts to first slow and then reverse the direction of the blast wind. Although the magnitude of the pressure in the negative phase is much less than that in the initial shock, its effects on a damaged structure can be quite significant.

For an observer $2 \mathrm{~m}$ from the centre of the charge and $50 \mathrm{~cm}$ above the ground, the ground reflection has overtaken the initial blast wave. The observer, only 'sees' the reflected wave. This is demonstrated in the pressure history at this point, Figure 9 (right). Notice that the negative phase is well established at $4 \mathrm{~ms}$ after detonation.

The above discussion has centred on a charge detonated above the ground. A charge detonated on the ground, will not produce a reflected shock or Mach stem. An observer will experience a blast wave characteristic of a free field charge. Thus the blast wave is a strong function of position and the obstacles in the path between the observer and the charge. Reflected waves can significantly increase the shock pressure and the blast wind. Thus an observer, depending on their position relative to the charge, may experience a blast wave that consists of a single shock wave, two shock waves or a single stronger reflected shock wave. Each scenario will also have an associated blast wind with it. It also explains why personnel close to a charge are often less seriously injured compared to those further away.

Having defined and explained the essential features of blast wave formation, propagation and development illustrated by the detonation of a $1 \mathrm{~kg}$ spherical charge of TNT, 
we can now consider how such a wave interacts with a structure.

\section{Interaction of a blast wave with a structure}

The blast wave from an explosion interacts with a structure by coupling energy from the blast flow field into the structure. This causes the structure to deform to an extent dependent upon the strength of the blast wave and the properties of the structure.

The characteristics of an explosive blast wave that have the most influence on the structural response are its peak pressure, impulse and overall shape. The strength and natural period of oscillation of the structure being loaded then determine the type of interaction and the response.

Unlike the case of the ground (considered a rigid surface) the blast wave impacting upon a structure will result in a shock wave being propagated through the structure as well as being reflected from it. The strength of the reflected and transmitted shock waves depends upon the material properties of the structure and its geometry and of course the incident blast wave. In addition, if the structure has internal spaces e.g. a building, or contains internal components e.g. the human chest, then the transmitted shock can induce quite dramatic and counter intuitive responses in these components.

When a blast wave strikes a structure the pressure loading that results is composed of two components. The first is the reflected shock pressure, which as described above can be significantly higher than the incident shock pressure. The second is the pressure developed by stopping or slowing down the blast wind.

To understand some of these effects it is useful to consider a simple pendulum, e.g. a swing or a weight on a string. Left to swing on its own it will have a natural frequency. If we try to force it to swing at a different frequency, then its response will depend on this forcing frequency. Frequencies close to its natural frequency will generate a large amplitude response in the pendulum. Frequencies far removed from its natural frequency will, however, generate a small amplitude response.

In addition, the time over which the force is applied i.e. the impulse, its direction and the point it is applied in the oscillation period, all control the finally observed amplitude. Thus a small force applied in phase with the pendulum can produce large amplitudes compared to a large force briefly applied out of phase with the pendulum.

A real structure, such as a building or a human, behaves in a very similar way, except that in this case the structure has to be represented by a large number of pendula each with a different natural frequency. The natural response of the structure is thus described by a number of modes of vibration each with a characteristic frequency. The response of the structure then depends upon how the blast wave couples energy into each of these modes of vibration.

We can also represent the blast wave as a series of pendula of different frequencies and amplitudes. As the blast wave develops and changes its shape, its natural frequency distribution will also change. Using this analogy we can begin to understand how energy is coupled to the matching modes of the structure and why the structural response will vary with distance from the explosion.

The overall shape of a blast wave may be qualitatively defined in terms of its rise time at the shock front, $T_{r}$ and the duration of the blast wind, $T_{d}$. The response then depends on how these characteristic times compare with the natural period of the structure $\mathrm{T}$.

There are four categories of response that are defined by this relationship:

Category $A$ : If the duration of the blast wave is short compared with the structures period of oscillation, the loading is partly absorbed by the structure's inertia, thus reducing the structural deformation.

Category B: If the duration of the blast wave is long compared with the structure's period of oscillation and has a long rise time, the structure experiences a load, which is effectively quasi-static. Here quasi-static means the pressure varies very slowly and could be approximated by an average constant value. The deformation is then the same as that produced by an equivalent static load.

Category $C$ : If the duration of the blast wave is long compared with the structure's period of oscillation and has a short rise time, then the structure experiences a deformation, which is greater than that caused by an equivalent static load.

Category D: If the duration of the blast wave is roughly equal to the structure's period of oscillation and the rise time is about half its duration, the structure's response may again exceed that caused by the equivalent static load.

One can now once again understand how distance from an explosion can alter the observed damage and why perversely objects and personnel close to an explosion often suffer less damage and injury than those further away from the explosion.

The relative importance of pressure and impulse in determining the structural response depends on whether the blast wave loading of the structure is 'impulsive' or 'quasi-static'.

Category $\mathrm{A}$ is termed impulsive, i.e. it is the impulse within the blast wave that determines the structure's response.

Categories B \& C are termed quasi-static loading, i.e. it is the pressure of the blast shock wave that determines the structure's response. 
Category $\mathrm{D}$ is part impulsive and part quasi-static and pressure and impulse determine the structure's response.

In general the sharper the rise time of the shock wave, the greater the structural response. Explosive blast waves with steep rise times are generated by high performance explosives such as TNT, HMX, RDX etc. Combustible gases such as hydrogen or liquids such as petrol generate explosive blast waves with shallow rise times.

From this discussion above it can be recognised that a given level of structural response or damage can result from either pressure, impulse or a combination of both. The actual mechanism will depend upon the explosion source and the distance from it.

This naturally leads to the very useful concept of the Iso-damage curve, the locus of explosions, which give the same structural response, or level of damage.

An example is shown in Figure 10. Points that lie on the left of the diagram (i.e. those with high impulse and low pressure) represent impulsive explosions and points that lie on the right of the curve (i.e. low impulse and high pressure) represent quasistatic explosions. Points in-between represent explosions, which are part impulsive and part quasi-static.

The curves predicting the probability of blast lung injury to personnel take this form.

\section{Internal damage mechanisms}

The discussion so far has assumed that the structure is represented by a single fundamental period of oscillation. As was pointed out above, many structures are complex and include components or internal structures that have very different oscillation periods. For example, the period of oscillation of the human thoracic wall is very different from that of say, alveolar septa, or solid viscera. Whilst the gross deformation of the external structure may load and compress these internal components, the transmitted shock from the incident blast wave can often cause more severe levels of damage. In many cases the material properties of the external structure can act to enhance these internal shock levels and hence damage.

To illustrate some of these effects, we return to our $1 \mathrm{~kg}$ spherical charge of TNT, detonated in free field, i.e. without the ground being present. We position two structures, diametrically opposite each other, $90 \mathrm{~cm}$ from the charge centre. The structures consist of $35 \mathrm{~cm}$ diameter spheres constructed from layers of materials. At the centre of each is a $10 \mathrm{~cm}$ diameter air space. This space is surrounded by a $10 \mathrm{~cm}$ thick layer of water, which in turn is surrounded, by a $8 \mathrm{~cm}$ layer of a material with a density $10 \%$ greater than the water ("unprotected"). The second structure, however, also has a $3 \mathrm{~cm}$ thick outer casing made from steel ("protected"). One could consider these objects to be constructed of biological materials. At this standoff from the charge the blast wave reaches the structures at about $300 \mu$ s, Figure 11.

Although the air shock (A) has separated from the product cloud (B), the products still make a significant contribution to the blast wave loading. When the blast wave strikes the structures it effectively 'wraps' itself around them. In our example this process is not completed until $900 \mu$ s after detonation, 600 $\mu$ s after the arrival of the blast wave, as shown in Figure 12. The product cloud has been slowed and deformed by its impact with the structures and is beginning to flow laterally (C). The impact of the products with the unprotected structure has also deformed and damaged it.

The figure graphically illustrates an additional feature of the interaction of a blast wave with a structure, namely its ability to

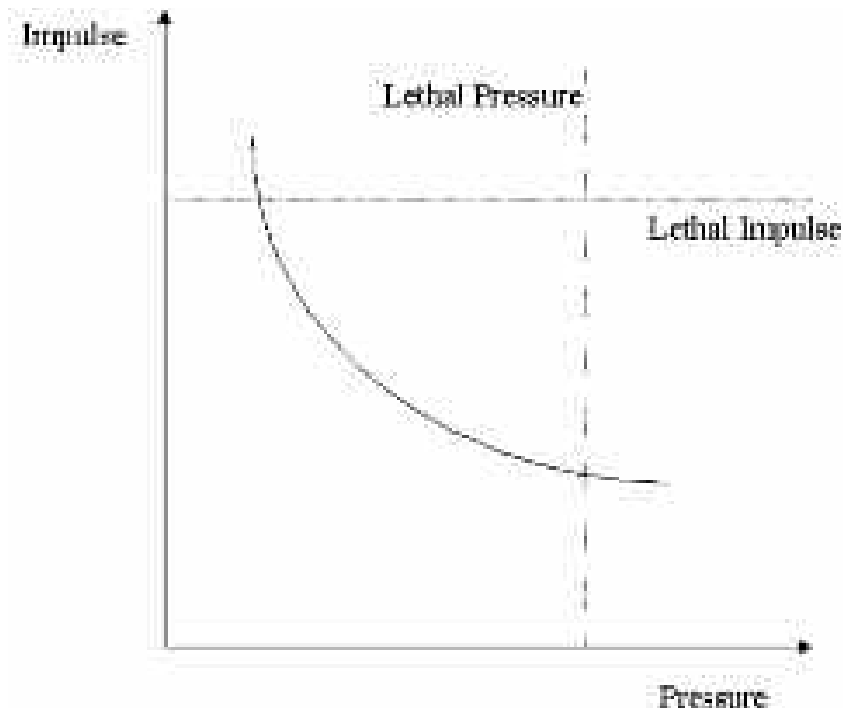

Fig 10. ISO-DAMAGE curve

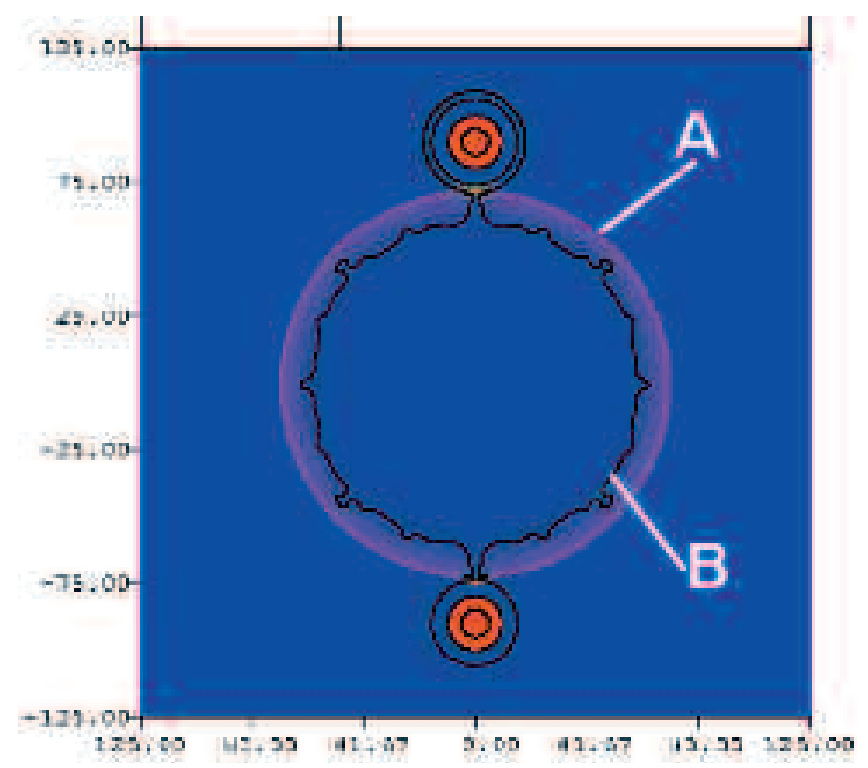

Fig 11. Initial Blast Wave Pressure Loading of unprotected (bottom) and protected (top) structures. 


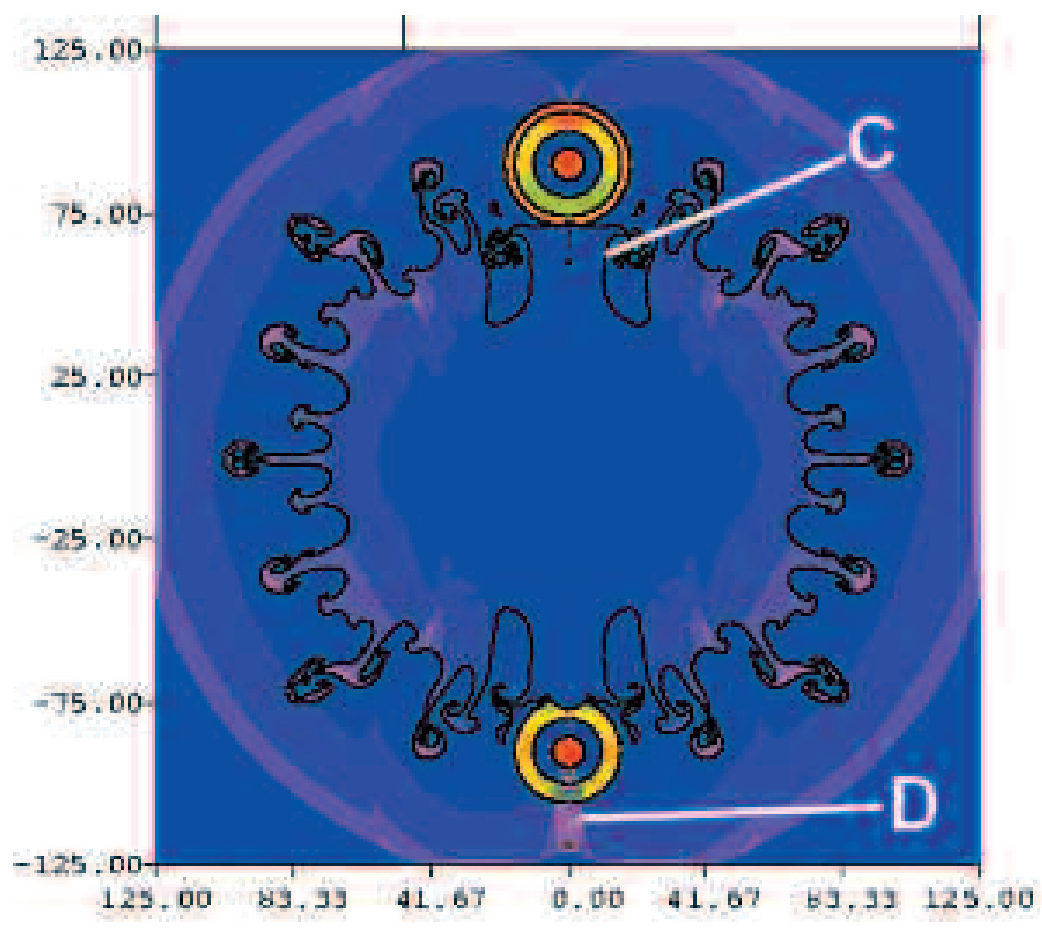

Fig 12. Blast Wave Pressure Loading at $900 \mu$ s of unprotected (bottom) and protected(top) structures. quite marked in the case of the protected structure. In the latter case there is also a marked spherical pressure gradient across the water layer, i.e. the water and the air space are being effectively squeezed.

In biological systems, this will lead to injuries such as bowel contusions.

The response of a structure is thus a complex problem. Intuitively obvious damage mechanisms, e.g. quasi-static pressure, in fact often do not represent the major cause of observed structural failure. The dynamic processes associated with material acceleration and high relative velocities are often far more effective. In the case of large structures, different parts may respond in different ways. It is therefore, vital that the important damage mechanisms are identified and understood.

In protecting a structure from blast, all possible damage mechanisms have to be considered and ranked. It is often the high frequency components induced by the blast wave that have to be effectively removed this is the case with primary blast lung injury. It must be recognised, however, that an inappropriate combination of materials in a protection scheme can enhance rather than dampen the damaging frequency components.

impact scenario, e.g. due to a projectile, where the loading is applied at the initial point of contact, a blast wave can load the rear of a structure long after the initial loading.

The reflected shock waves generated by the structures can be seen propagating away from them in Figure 12 at $900 \mu \mathrm{s}$. They modify the incident blast field. Observers close to the structures will therefore, experience a complex pressure loading and blast wind. Furthermore, when the shock waves enveloping the structure, meet at the opposite pole there is a strong pressure enhancement, because the flow is effectively stagnated, Figure 12 (D). The resultant loading in the structures can also be observed in the figure.

In terms of the internal response, the different material speeds of sound imply that the shock waves developed by the impact of the blast wave propagate at different velocities through them. The protected structure, because of the steel case, produces a stronger reflected blast wave. The confinement also results in higher and more uniform pressures within the internal components.

Internal elements of the structure will respond differently to these effects. This can lead to relative motion between them with consequent shearing and tearing at interfaces. Most materials, including biological materials, are relatively weak in shear and therefore readily fail under shear loading conditions.

The pressure field shown in the Figure 12 highlights high-pressure regions at the opposite poles of both spherical structures,

\section{Conclusions}

This paper has described how an explosion due to a sudden release of energy forms blast waves. The blast wave is composed of two parts, the initial shock wave and the blast wind. The shock wave results when we try to drive a disturbance supersonically in a medium. A shock wave modifies the properties of the medium through which it is travelling. The resulting non-linear behaviour means that reflection of a shock wave is also non-linear. Significant pressure enhancements can be generated on reflection and for angles above $40^{\circ}$ a Mach stem is produced.

The detonation of a $1 \mathrm{~kg}$ spherical charge of TNT has been simulated, using a Eulerian hydrocode cAst, to illustrate the formation, development and propagation of blast waves. Numerical simulation is a powerful tool for understanding blast waves and their interaction with a structure.

The interaction of the blast wave with the ground has been shown to lead to its significant enhancement. An observer positioned at different distances from the explosion will experience very different blast waves, with characteristically different pressures, blast winds and durations.

The interaction of a blast wave with a structure has been shown to be a complex process that is dependent upon its natural frequencies. The subsequent response can be impulsive, quasi-static or a combination of both, dependent upon the explosion source and the stand off distance. The same level of 
damage can, therefore, be created by different loading regimes. This naturally leads to the concept of an iso-damage curve for a structure.

The phenomena associated with the interaction of a blast wave with a structure have been illustrated with a simple hydrocode simulation. The ability of a blast wave to load all parts of a structure has been shown to be crucial in describing the overall structural response. An understanding of the damage mechanisms is important if vulnerability and protection of a structure, particularly personnel, is required.

Simple intuitive ideas on structural protection can lead to significantly increased levels of damage. This occurs because high frequency components of the loading are often enhanced by an inappropriate selection of materials. 